\title{
Georegistration of Earth Observing-1 (EO-1) Data Using Global Land Survey (GLS) Maps
}

Jacqueline Le Moigne ${ }^{1}$, Patricia Sazama ${ }^{1 \& 2}$, Steve Swanson ${ }^{3}$, Vuong Ly ${ }^{1}$ and Dan Mandl ${ }^{1}$

1. NASA Goddard Space Flight Center, Software Engineering Division

2. University of Maryland, Computer Science Department

3. Princeton University, Computer Science Department 


\section{Background - Image Registration}

- What is Image Registration?

"Exact pixel-to-pixel matching of two different images or matching of one image to a map"

- Navigation or Model-Based Systematic Correction

- Orbital, Attitude, Platform/Sensor Geometric Relationship, Sensor Characteristics, Earth Model, etc.

- Image Registration/Feature-Based Precision Correction

- Navigation within a Few Pixels Accuracy

- Image Registration Using Selected Features (or Control Points) to Refine Geo-Location Accuracy

- Image Registration as a Post-Processing or as a Feedback to Navigation Model 


\section{Image Registration Frameworks}

- Mathematical Framework

- I1(x,y) and I2(x,y): images or image/map

- find the mapping (f,g) which transforms I1 into I2:

$$
\begin{aligned}
& \mathbf{I 2}(\mathbf{x}, \mathbf{y})=\mathrm{g}(\mathbf{I} 1(\mathbf{f x}(\mathbf{x}, \mathbf{y}), \mathbf{f y}(\mathbf{x}, \mathbf{y})) \\
& \text { "f : spatial mapping } \\
& \text { " g: radiometric mapping }
\end{aligned}
$$

- Spatial Transformations "f"

- Translation, Rigid, Affine, Projective, Perspective, Polynomial, ...

- Radiometric Transformations "g" (Resampling)

- Nearest Neighbor, Bilinear, Cubic Convolution, ...

- Algorithmic Framework (Brown, 1992)

1. Feature Extraction

2. Feature Matching (Similarity Metrics \& Matching Strategy)

3. Image Resampling (if needed) 


\section{Image Registration Components}

0 Pre-Processing

- Cloud Detection, Region of Interest Masking, ...

1 Feature Extraction ("Control Points")

- Gray Levels, Salient Points (e.g., Edges, Edge-like such as Wavelet Coefficients, Corners), Lines, Contours, Regions, Scale Invariant Feature Transform (SIFT), etc.

2 Feature Matching

- Choice of Spatial Transformation (function f: a-priori knowledge)

- Choice of Search Strategy :

- Global vs Local, Multi-Resolution, Optimization, ...

- Choice of Similarity Metrics

- L2-Norm, Normalized Cross-Correlation, Mutual Information, Hausdorff Distance, ...

3 Remapping/Resampling (function g: if necessary) 


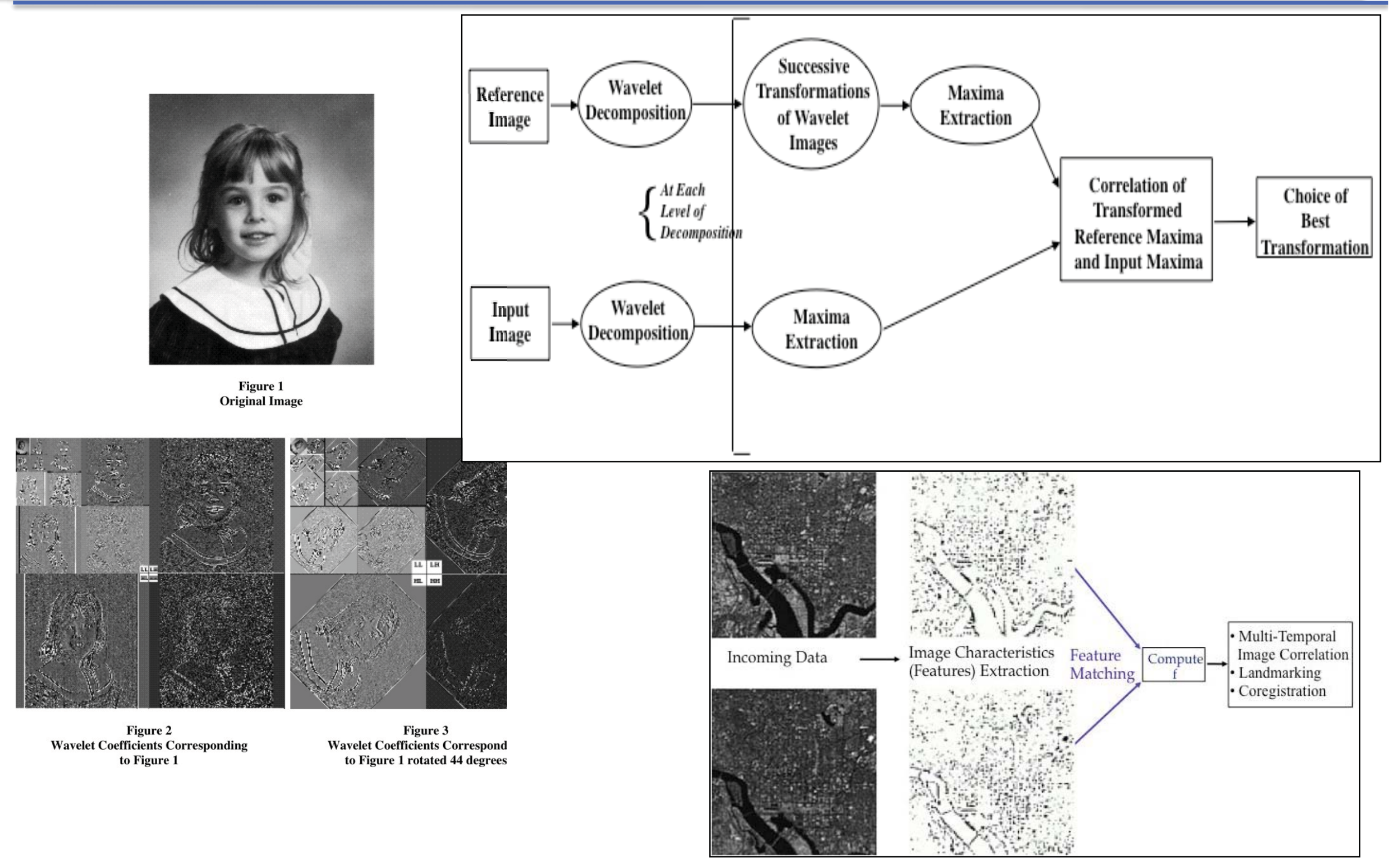




\section{Rotation- and Translation-Invariant Representations}

- Spline Wavelets [Battle \& Lemarié; Unser et al]

$$
\begin{aligned}
& V_{i}^{n}=\left\{g_{i}^{n}(x)=\sum_{k=-\infty}^{+\infty} c_{i}(k) \varphi^{n}\left(2^{-i} x-k\right), x \in \mathfrak{R}, c_{i} \in l_{2}\right\} \\
& \text { with scaling function } \varphi^{n}(x)=\sum^{+\infty} p(k) \beta^{n}(x-k) \\
& p \text { arbitrary invertible convolutión } \bar{n}^{\infty} \text { operator or filter, } \\
& \text { and } \beta^{n}(x) \text { is a } B \text {-spline of order } n \text { (can be } \\
& \text { constructed by repeated convolution of } B \text {-Spline } \\
& \text { of order } 0)
\end{aligned}
$$

Example of B-Spline Scaling Function and Associated Wavelet

- Simoncelli et al

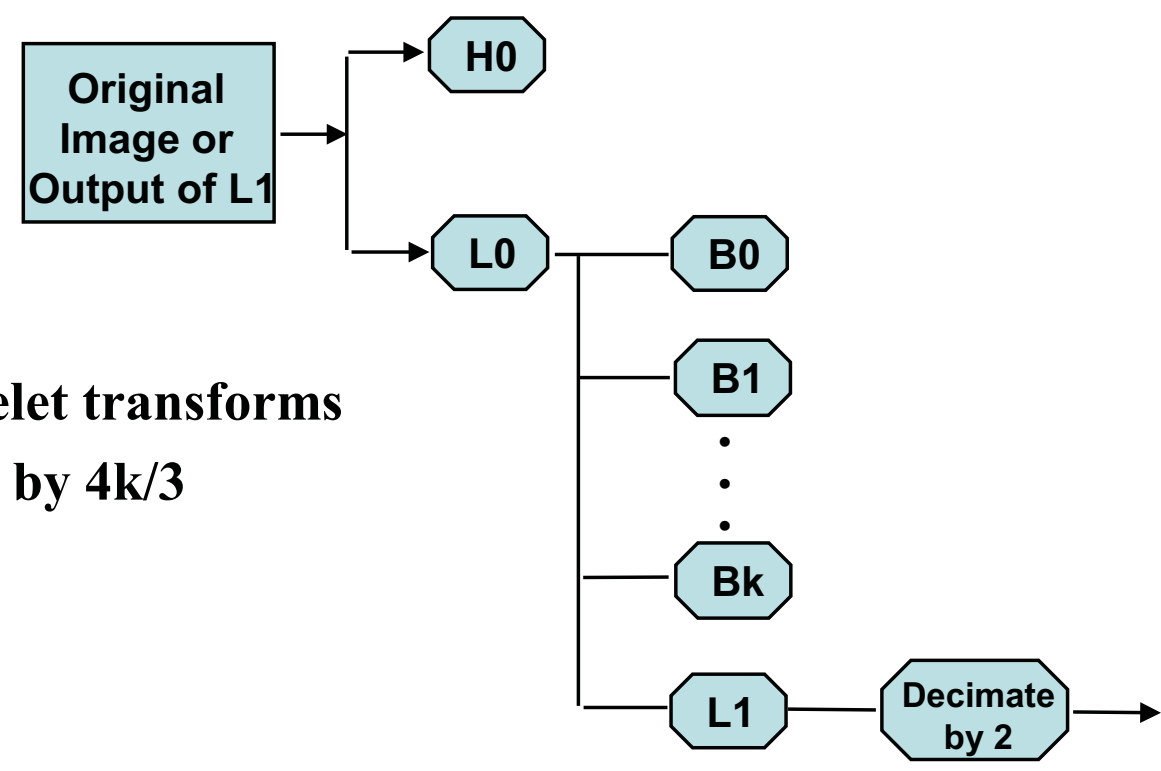

- Relax critical sampling condition of wavelet transforms

- Provides an overcomplete representation by $4 \mathrm{k} / 3$ 


\section{Matching Strategies}

- Exhaustive Search

- Fast Fourier Transform

- Optimizations:

- Gradient Descent $\left.\left.\left\lceil\sum f_{x}^{2} \quad \sum f_{x} f_{y} \quad \sum R f_{x}\right\rceil \Delta x\right\rceil \quad \sum \sum(f-g) f_{x}\right\rceil$

$$
\left.\mid \begin{array}{lll}
\sum f_{x} f_{y} & \sum f_{x}^{2} & \sum R f_{y} \| \Delta y|=| \sum(f-g) f_{y} \mid \\
\sum R f_{x} & \sum R f_{y} & \left.\left.\sum R^{2}\right\rfloor \Delta \theta\right\rfloor \mid\lfloor(f-g) R
\end{array}\right]
$$

- Modified Marquart-Levenberg: hybrid optimization approach between a pure gradient-descent approach and a more powerful but less robust Gauss-Newton method, implemented in a multi-resolution fashion

- Spall's Simultaneous Perturbation Stocchastic Approximation (SPSA): based on gradient approximation computed from objective function (200 iterations)

- Robust Feature Matching

- Hierarchical Subdivisions of Search Space

- Pruning of Search Space 


\section{Matching Strategies}

- Exhaustive Search

- Fast Fourier Transform

- Optimizations:

- Gradient Descent $\left.\left.\left\lceil\sum f_{x}^{2} \quad \sum f_{x} f_{y} \quad \sum R f_{x}\right\rceil \Delta x\right\rceil \quad \sum \sum(f-g) f_{x}\right\rceil$

$$
\left|\begin{array}{lll}
\sum f_{x} f_{y} & \sum f_{x}^{2} & \sum R f_{y}
\end{array} \|_{\Delta y}\right|=\left|\sum(f-g) f_{y}\right|
$$

- Modified Marquart-Levenberg: hybrid optimization approach between a pure gradient-descent approach and a more powerful but less robust Gauss-Newton method, implemented in a multi-resolution fashion

- Spall's Simultaneous Perturbation Stocchastic Approximation (SPSA): based on gradient approximation computed from objective function (200 iterations)

- Robust Feature Matching

- Hierarchical Subdivisions of Search Space

- Pruning of Search Space 


\section{Global Land Survey (GLS) Maps}

- A collection of Landsat-type satellite images from USGS

- Near complete global coverage

- Orthorectified

- Each image has cloud cover of less than 10\%

- Four versions: 1970, 1990, 2000 and 2005

- Current Ground Truth or "Reference Chips" extracted from the GLS 2000 (can be updated when the GLS 2010 is completed)

- Reference Chips of size 256 X 256

- http://landsat.usgs.gov/science GLS.php 


\section{Chip Registration}

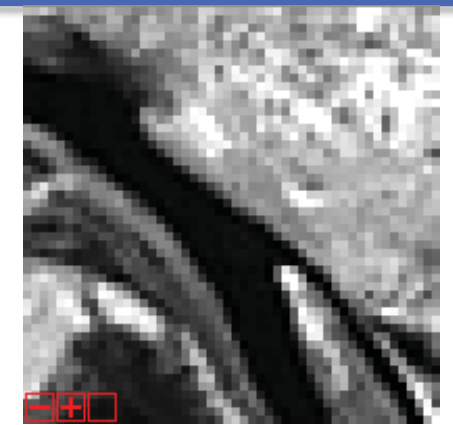

Overlapping chip from database

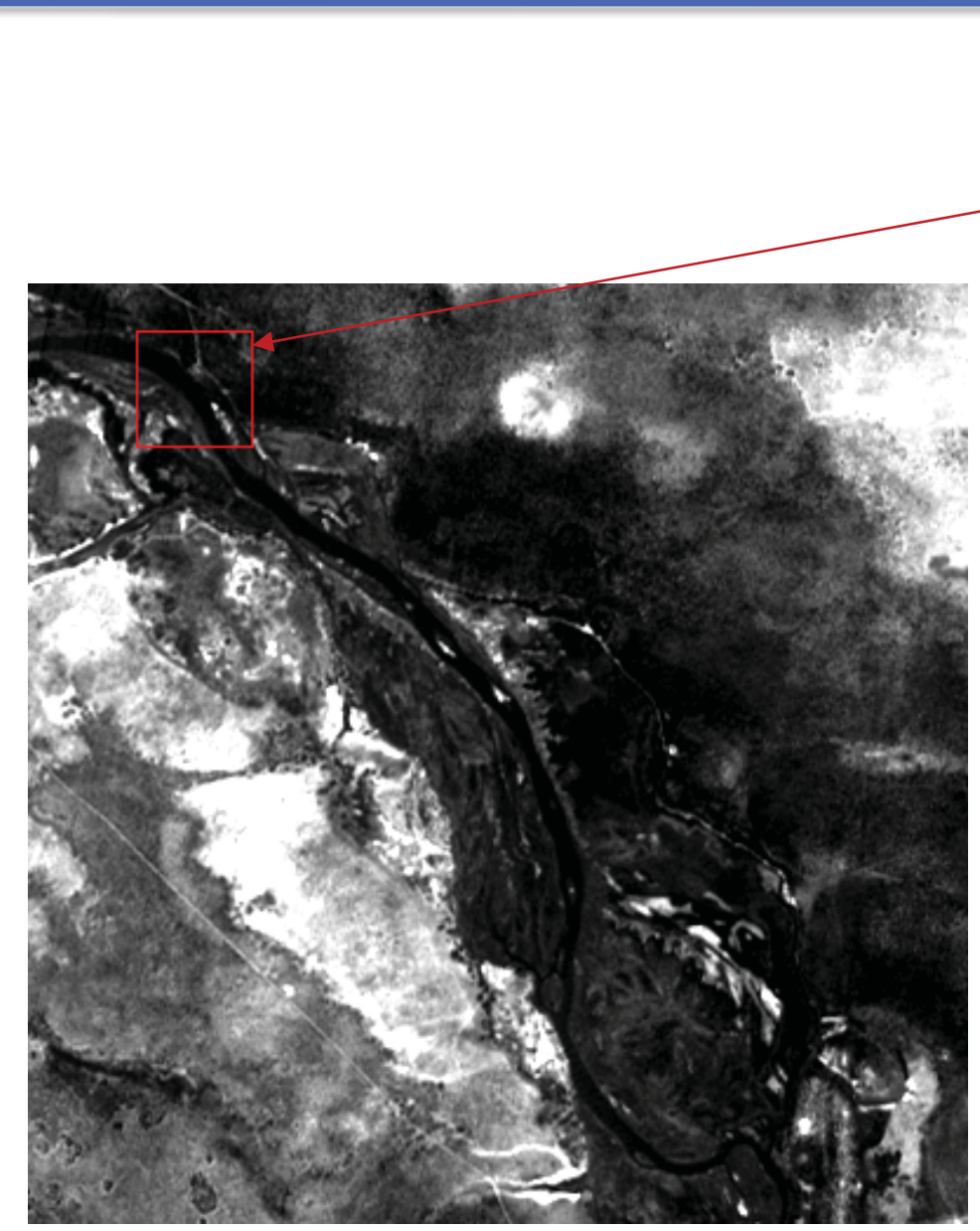

Area in E01 scene where chip was extracted

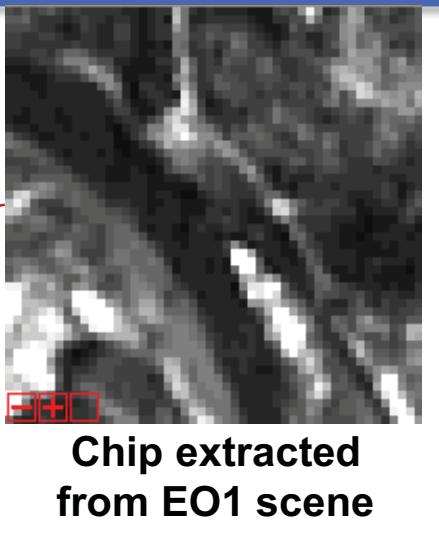

Currently "chip database" created (in a brute-force fashion) by extracting successive $256 x 256$ sub-images of all GLS scenes and storing them according to path and row 


\section{Automatic Registration of EO-1 Scenes Using Global Land Survey (GLS) Database}
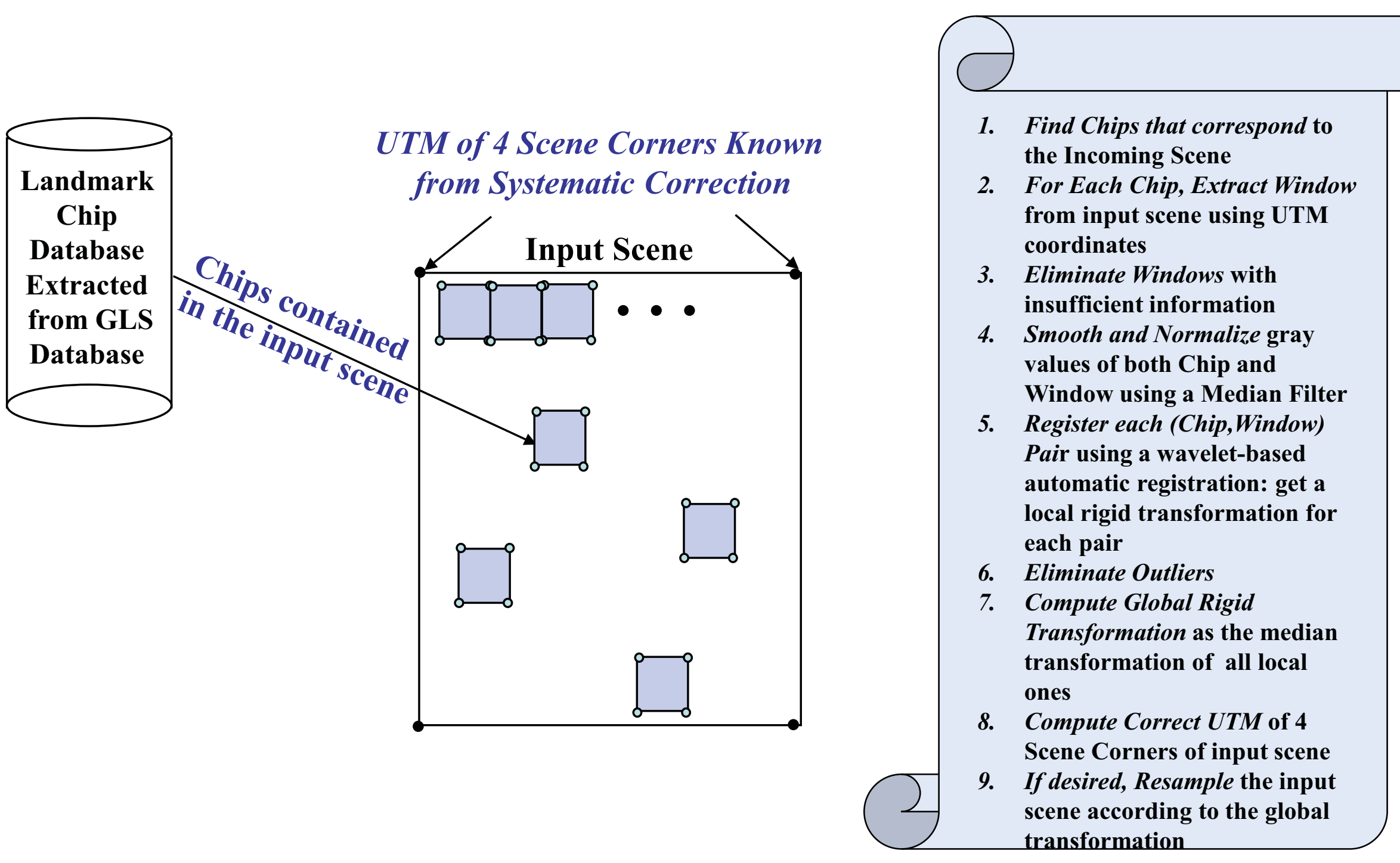
Scene 1 Before Automatic Registration Superimposed onto Google Earth

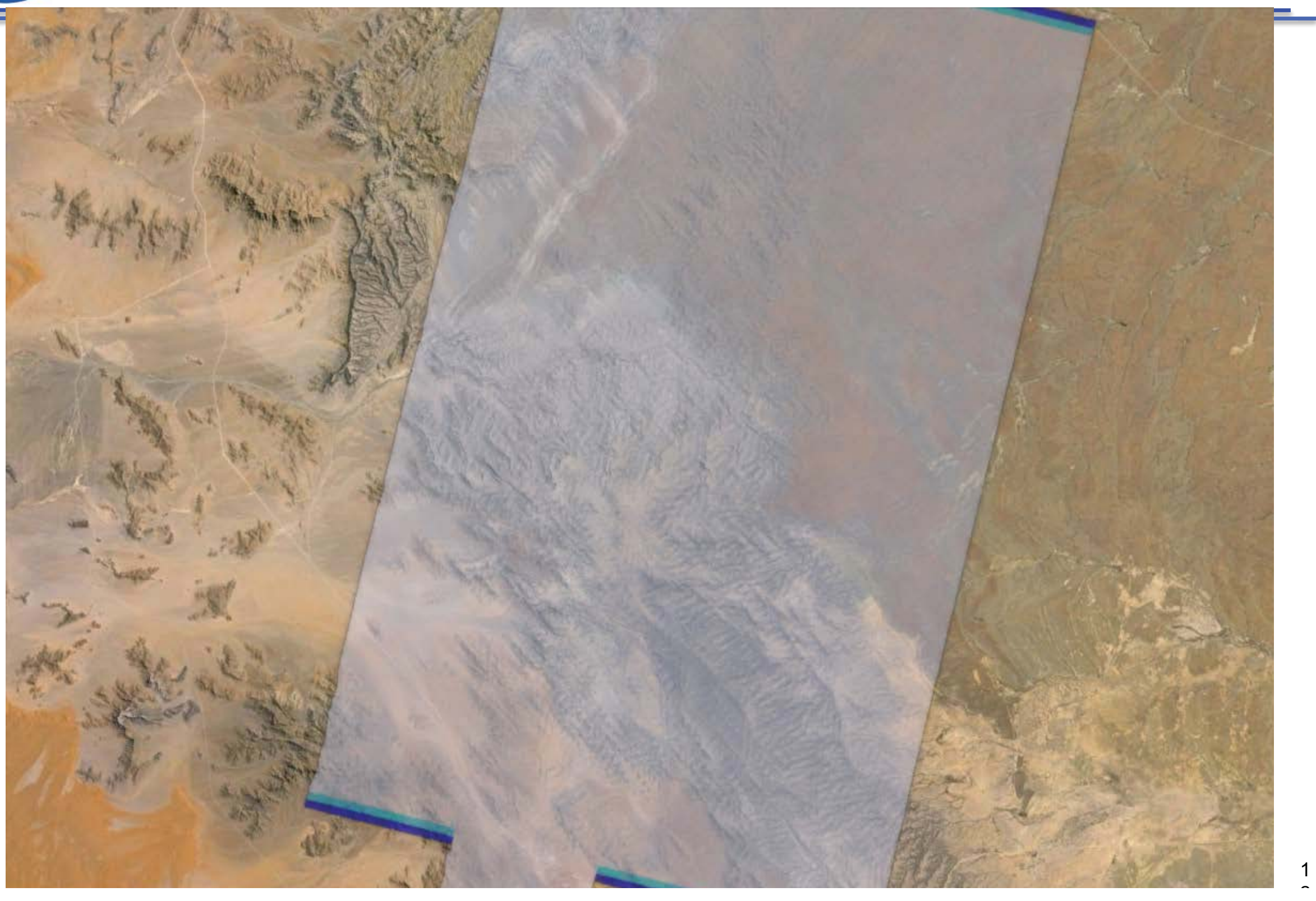


Scene 1 After Automatic Registration NASA

\section{Superimposed onto Google Earth}

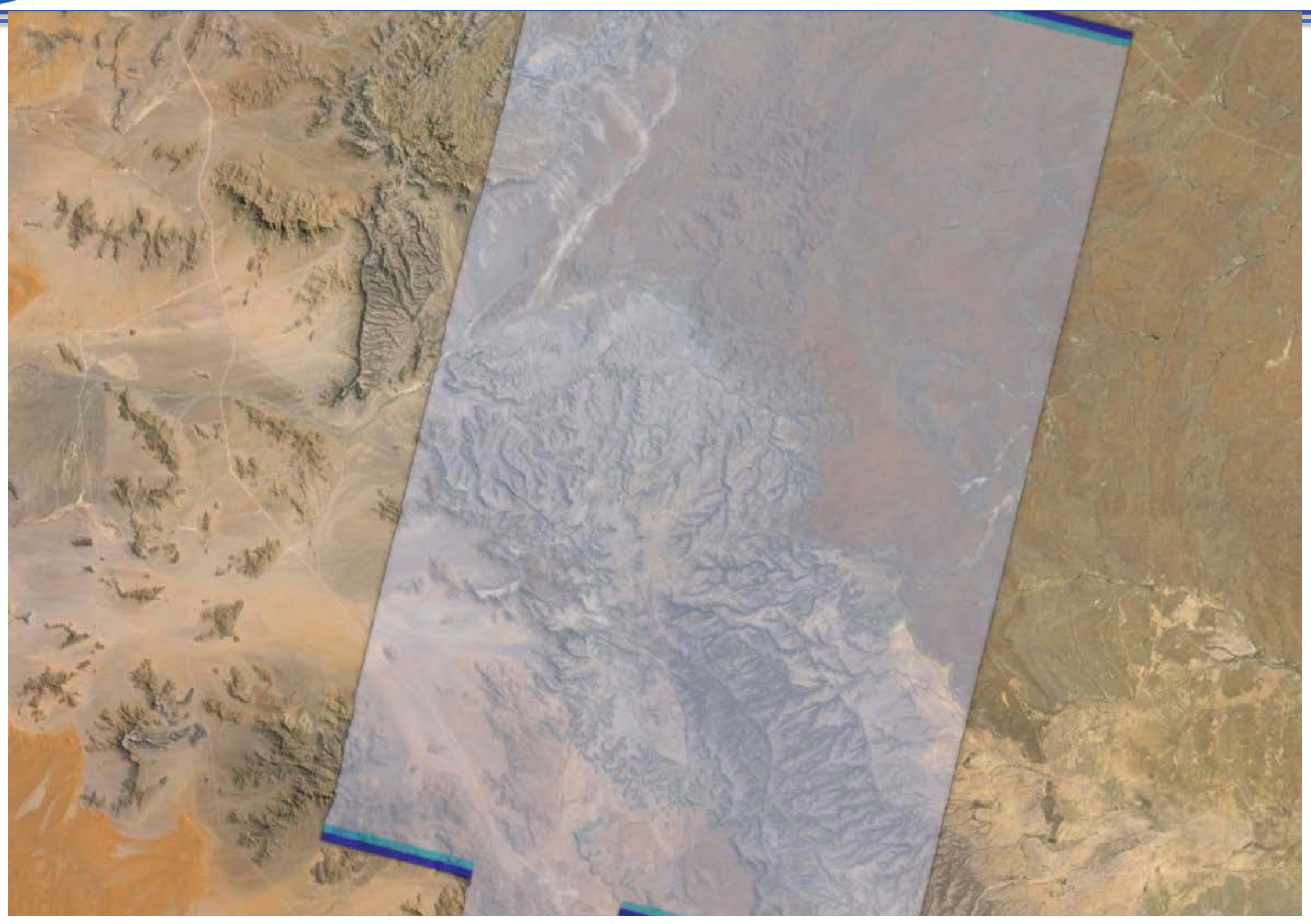


Scene 2 Before Automatic Registration Superimposed onto Google Earth

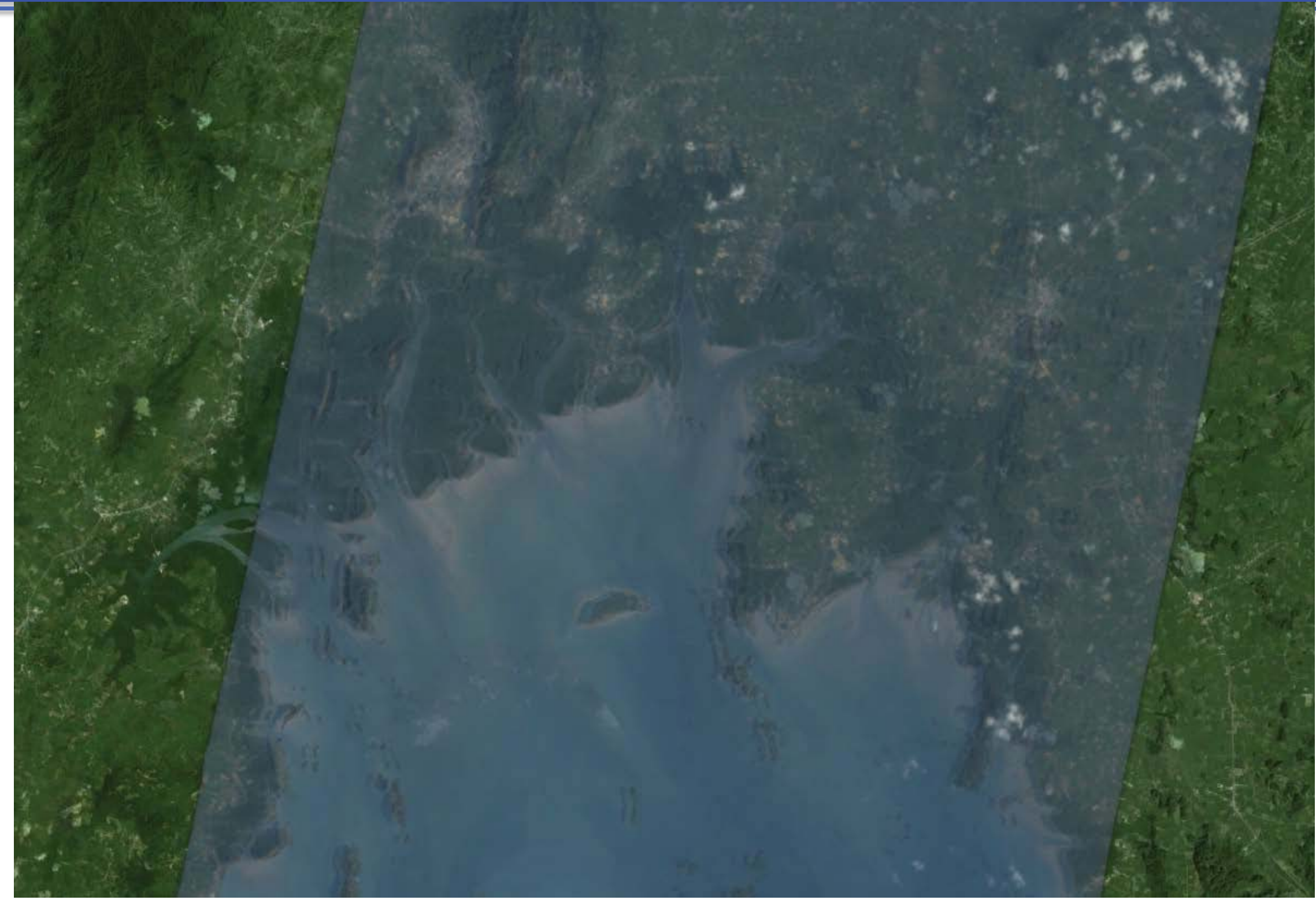


Scene 2 After Automatic Registration Superimposed onto Google Earth

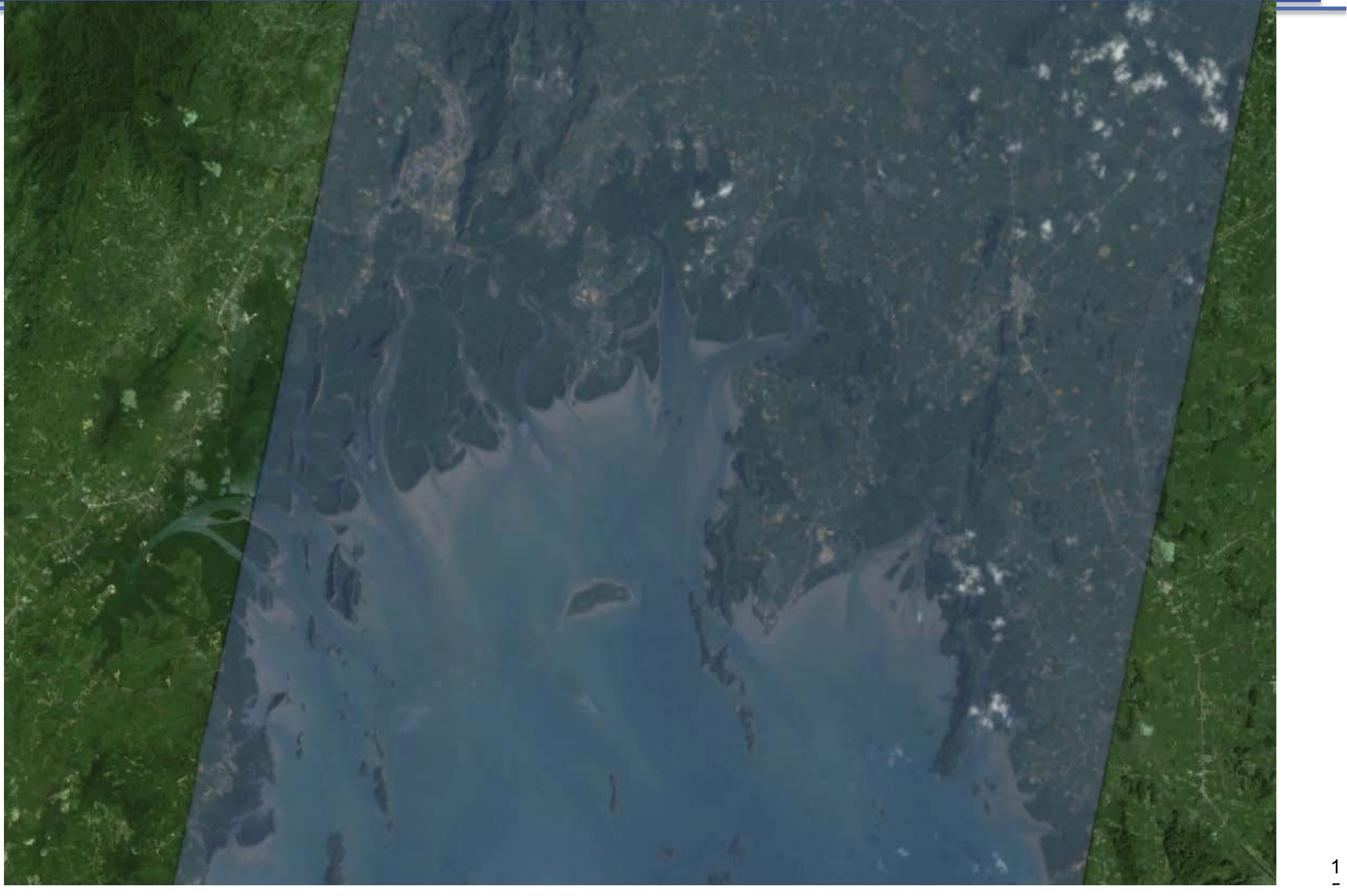




\section{Quantitative Results With All Chips ("Wall-to-Wall)}

- Scene 1 (EO1A1780772013325110KF)

$\circ \quad$ Wavelet Registration (Median Global Transformation, after outlier elimination)

$$
\mathrm{Tx}=-15.84, \mathrm{Ty}=-18.17 \text {, Theta }=-0.0083, \text { Scale }=1.0
$$

○ Manual registration (using ENVI):

$\mathrm{Tx}=-15.99, \mathrm{Ty}=-20.49$, Theta $=0.0224$, Scale $=1.0$

$\circ \quad$ Error in $(T x, T y, T h e t a)=(0.15,2.32,0.03)$

- Scene 2 (EO1A1300542014053110PZ)

- Wavelet Registration (Median Global Transformation, after outlier elimination)

$$
\mathrm{Tx}=-14.32, \mathrm{Ty}=-3.12, \text { Theta }=-0.0211, \text { Scale }=1.0
$$

○ Manual registration (using ENVI):

$$
\mathrm{Tx}=-16.45, \mathrm{Ty}=-4.99, \text { Theta }=0.0218, \text { Scale }=1.0
$$

$\bigcirc \quad$ Error in $(\mathrm{Tx}, \mathrm{Ty}, \mathrm{Theta})=(2.13,1.87,0.04)$

TIMING - Running Python Script : 19.36s 


\section{Chips Selection Using Entropy}

- If Chips pre-selected based on the information content (e.g., using an entropy measure)

$\Rightarrow$ Registration may be more accurate because transformation only computed on pairs that have a significant amount of features

$\Rightarrow$ Registration faster because less local registrations

$\Rightarrow$ Chip database smaller to be stored onboard

- Compute Entropy of all Chips Using Histogram:

$$
H=-\sum_{i=0}^{255} p_{i} \log p_{i} \quad \begin{aligned}
& \text { where } p_{i} \text { is the value of the histogram } \\
& \text { for gray value } i
\end{aligned}
$$

- Keep only Chips with Entropy Above Threshold

- $\quad$ Number of Chips Scene 1/Scene 2:

- Before Selection:

- $\quad$ After Entropy Selection: 


\section{Quantitative Results Only Keeping Chips with High Entropy}

- Scene 1 (EO1A1780772013325110KF)

$\circ \quad$ Wavelet Registration (Median Global Transformation, after outlier elimination)

$$
\mathrm{Tx}=, \mathrm{Ty}=\text {, Theta }=\text {, Scale }=1.0
$$

- Manual registration (using ENVI):

$\mathrm{Tx}=-15.99, \mathrm{Ty}=-20.49$, Theta $=0.0224$, Scale $=1.0$

$\circ \quad$ Error in $(\mathbf{T x}, \mathbf{T y}, \mathbf{T h e t a})=(,, \quad)$

- Scene 2 (EO1A1300542014053110PZ)

- Wavelet Registration (Median Global Transformation, after outlier elimination)

$$
\mathrm{Tx}=, \mathrm{Ty}=\text {, Theta }=, \text { Scale }=1.0
$$

$\circ \quad$ Manual registration (using ENVI):

$$
\mathrm{Tx}=-16.45, \mathrm{Ty}=-4.99, \text { Theta }=0.0218, \text { Scale }=1.0
$$

$\circ \quad$ Error in $(\mathbf{T x}, \mathbf{T y}$, Theta $)=(,$, 


\section{Conclusions and Future Work}

- Results visually acceptable with fast and real-time computations

- Applicable on the ground or on-board

- Computations can be made more accurate and faster by pre-selecting the chips for information content:

- Initial experiments using entropy $=>$ better accuracy and faster computations

- Potential future improvements:

- Investigate other chip pre-selection methods, e.g., edgeness count, land cover classification, etc.

- Use information content method also on extracted windows to only register pairs with sufficient information content

- Other Improvements:

- Compute global transformation from the list of corners coordinates (GP's)

$=>$ after outlier elimination, compute rigid, affine or polynomial transformation

- Include cloud and water masks

- Implement automatic chip registration on SpaceCube or hybrid processor

- If no database onboard, incorporate automatic "region of interest extraction"

$=>$ change detection can be performed onboard without chip database 